\title{
Lateralized spatial and object memory encoding in entorhinal and perirhinal cortices
}

\author{
Patrick S.F. Bellgowan, ${ }^{1,5,6}$ Elizabeth A. Buffalo, ${ }^{2,3}$ Jerzy Bodurka, ${ }^{4}$ and Alex Martin ${ }^{1}$ \\ ${ }^{1}$ Section on Cognitive Neuropsychology, NIMH, Bethesda, Maryland 20892, USA; ${ }^{2}$ Yerkes National Primate Research Center, \\ Atlanta, Georgia 30329, USA; ${ }^{3}$ Department of Neurology, Emory University School of Medicine, Atlanta, Georgia 30329, USA; \\ ${ }^{4}$ Functional MRI Facility, NIMH, Bethesda, Maryland 20892, USA
}

\begin{abstract}
The perirhinal and entorhinal cortices are critical components of the medial temporal lobe (MTL) declarative memory system. Study of their specific functions using blood oxygenation level-dependent (BOLD) functional magnetic resonance imaging (fMRI), however, has suffered from severe magnetic susceptibility signal dropout resulting in poor temporal signal-to-noise (tSNR) and thus weak BOLD signal detectability. We have demonstrated that higher spatial resolution in the $z$-plane leads to improved BOLD fMRI signal quality in the anterior medial temporal lobes when using a 16-element surface coil array at $3 \mathrm{~T}$ (Tesla). Using this technique, the present study investigated the roles of the anterior medial temporal lobe, particularly the entorhinal and perirhinal cortices, in both object and spatial memory. Participants viewed a series of fractal images and were instructed to encode either the object's identity or location. Object and spatial recognition memory were tested after 18-sec delays. Both the perirhinal and entorhinal cortices were active during the object and spatial encoding tasks. In both regions, object encoding was biased to the left hemisphere, whereas spatial encoding was biased to the right. A similar hemispheric bias was evident for recognition memory. Recent animal studies suggest functional dissociations among regions of the entorhinal cortex for spatial vs. object processing. Our findings suggest that this process-specific distinction may be expressed in the human brain as a hemispheric division of labor.
\end{abstract}

Studies in rodents and monkeys are suggestive of a process-specific division of labor within the perirhinal and entorhinal cortices. For example, electrophysiological and lesion studies in rodents show that within the entorhinal cortex more medial subdivisions receive and process spatial information, whereas lateral regions receive and process object-related information (Fyhn et al. 2004; Hafting et al. 2005; Hargreaves et al. 2005; Seffenach et al. 2005; Kerr et al. 2007).

Correspondingly, in the monkey, the dorsal and ventral visual processing streams have different projection paths along the rostro-caudal extent of the parahippocampal cortex, perirhinal, entorhinal cortex and throughout the perforant pathway to the hippocampus (Suzuki and Amaral 1994, 2004; Suzuki et al. 1997; Burwell 2000; Munoz and Insausti 2005). Superimposed upon this rostro-caudal topography are medial to lateral afferent projection gradients within both perirhinal and entorhinal cortices (Saleem and Tanaka 1996; Mohedano-Moriano et al. 2007). Although functional dissociations between the medial and lateral regions of these cortical structures have yet to be demonstrated, the anatomical connectivity is suggestive of a medial to lateral functional distinction in the monkey similar to that found in rodents.

In humans, both neuropsychological (e.g., Kimura 1963; Milner 1972; Glosser et al. 1995; Jones-Gotman et al. 1997; Bohbot et al. 2000; Kelley et al. 2002; Kennepohl et al. 2007) and neuroimaging (e.g., Moscovitch et al. 1995; Martin et al. 1997; Bellgowan et al. 1998; Kelley et al. 1998; Sommer et al. 2005) studies have documented material and process-specific distinctions in the medial temporal lobe (MTL). In contrast to the ani-

\footnotetext{
${ }^{5}$ Present address: Laureate Institute for Brain Research, Tulsa, OK 74133, USA.

${ }^{6}$ Corresponding author.

E-mail pfbellgowan@saintfrancis.com; fax (918) 491-5792.

Article is online at http://www.learnmem.org/cgi/doi/10.1101/lm.1357309.

Freely available online through the Learning \& Memory Open Access option.
}

mal studies, however, these reports indicate that human MTL functional dissociations are primarily associated with differences in hemispheric laterality, with left MTL processing meaningful or linguistic information and right MTL processing spatial information.

In addition to these lateralized differences, distinctions in human memory function have also been reported that follow proposed connectivity to spatial and object processing regions. Specifically, we recently reported that the parahippocampal cortex, which is known to receive greater input from spatial processing areas (posterior parietal and retrosplenial cortex) than object processing areas (ventral occipitotemporal cortex), showed preferential processing of spatial over object information (Buffalo et al. 2006). We also observed activity in perirhinal cortex for both object and spatial encoding. However, neither within region nor lateralized functional distinctions was observed. Interpretation of these negative topographical findings must be tempered by the fact that low signal-to-noise ratio (SNR) and high signal dropout have plagued neuroimaging studies of perirhinal and entorhinal cortices. To combat this problem, we used multichannel imaging at higher spatial resolution to enhance the temporal signal-tonoise ratio (tSNR) in the anterior MTL and improve signal detection of both entorhinal and perirhinal cortices (Bellgowan et al. 2006).

Improved imaging of these regions allows us to evaluate two important questions regarding functional dissociations within the MTL. First, do perirhinal and entorhinal cortices make unique contributions to memory encoding and recognition of object and spatial information? Second, is there a hemispheric division of labor in these structures as suggested by neuropsychological investigations?

Figure 1A depicts the behavioral paradigm used in the current study. In a blocked design, Object and Spatial memory tasks that included Encoding, Post-Encoding, Recognition, and 
A

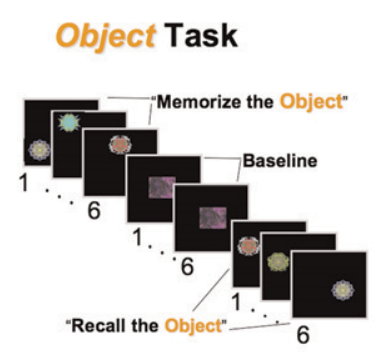

Spatia/ Task

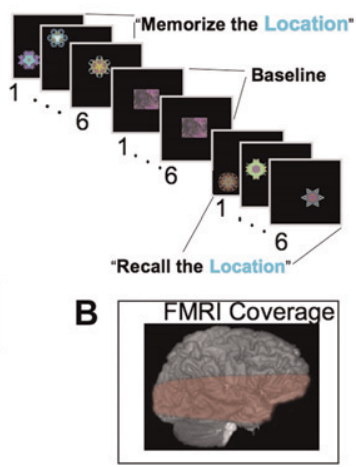

Figure 1. (A) Methods for both the Object and Spatial memory tasks used during fMRI scanning. The numbers below each image are TR units $(3 \mathrm{sec})$ representing the length of time each image was presented to the subject. The instructions that were provided for each block are presented in quotation marks. (B) A representative fMRI imaging volume covering the majority of the temporal lobe.

Post-Recognition phases were alternated. During the Encoding and Recognition phases of both tasks, a sequence of six fractal images was presented. The Object memory task required memory and later recognition of specific fractal images regardless of location, whereas the Spatial task required encoding and later recognition of the location in which the fractals were presented.

\section{Results}

\section{Behavioral data}

Recognition memory performance did not differ between tasks based on percent correct (78\% Object; $75 \%$ Spatial), or $d^{\prime}$ (Object $d^{\prime}=2.14$; Spatial $d^{\prime}=2.06$ )

\section{Imaging data}

Results of the group ANOVA for voxel clusters passing a corrected $P<0.05$ threshold are summarized in Table 1. Anterior MTL structures including the anterior parahippocampal cortex, entorhinal, and perirhinal cortices all showed activation for the main effects of Task and Phase and for the interaction of Task $\times$ Phase. The directions of these effects were then investigated using the simple main effects and a priori means contrast maps. The brain image in Figure 2 depicts the simple main effect for Encoding (both encoding tasks vs. baseline) using the Mixed-Effects ANOVA thresholded at a corrected $P<0.05$. This figure reveals significant activation in the hippocampus and MTL cortical regions including the perirhinal and entorhinal cortices. The Encoding Simple Main Effect map could represent either both encoding tasks greater than the scrambled baseline or either encoding task greater than scrambled baseline. To both determine the contribution of each encoding task to the overall Encoding Simple Main Effect and provide an estimate of the shape of the blood oxygenation leveldependent (BOLD) response during each task, estimated impulse response functions (IRF) were evaluated using voxels within the hippocampus, entorhinal, and perirhinal cortices that showed significant Encoding effects. The IRFs from these voxels were then extracted for both the Object and Spatial encoding tasks to show the strength of this effect for each task. The hippocampus, entorhinal, and perirhinal cortices all show increased BOLD responses for both the Object and Spatial encoding tasks (all $P \mathrm{~s}<$ 0.05, corrected; for each task vs. baseline, see Fig. 2).

Maps of the a priori group contrast of means between the Object and Spatial tasks during both Encoding (Fig. 3A) and
Recognition (Fig. 3B) are presented in Figure 3. Results are consistent with Buffalo et al. (2006) showing preferential activation during Object Encoding in posterior regions of the parahippocampal cortex with an anterior region of the parahippocampal cortex, just posterior to the perirhinal cortex, showing greater activation during Spatial Encoding. Anterior temporal regions including the perirhinal and entorhinal cortex show biased lateralized activity with more left activity for Object Encoding and more right hemisphere activity for Spatial Encoding. Additionally, both the amygdala and anterior hippocampus show differential encoding-related activity. The amygdala activity was limited to encoding of Objects with no significant activity for Spatial Encoding. Hippocampus activity showed preferential anterior hippocampal activity for Object Encoding compared to Spatial Encoding.

Analysis of the Recognition-related activity showed no clusters of activation surviving the multiple comparisons correction for the contrast between Object and Spatial Recognition in the entorhinal or perirhinal cortex. This null result may reflect the high level of anatomical intersubject variability that can be detrimental to detecting spatial activation patterns when standard stereotaxic registration is performed (Miller et al. 2005). To further explore Recognition-related activity, we created individual subject maps of the Object vs. Spatial Recognition conditions (Fig. 4). Statistically corrected individual maps (see Fig. 4) show that within individuals, both the entorhinal and perirhinal cortex participate in Object and Spatial Recognition. Activation maps in Figure 4 have been masked so as to only show activation surviving multiple comparisons correction in the entorhinal and perirhinal cortex.

To quantify the degree of lateralization, we tabulated laterality indices (see Materials and Methods) for the Object vs. Spatial Encoding contrast (see Fig. 5A,B). ANOVA performed on the nontabulated laterality volume measures showed a significant interaction of Task by Hemisphere $\left(F_{(1,13)}=7.143 ; P<0.05\right)$ with more active tissue in the left hemisphere during Object Encoding $\left(t_{(1,13)}=5.176 ; P<0.05\right)$ and more voxels in the right hemisphere active for spatial encoding $\left(t_{(1,13)}=4.684 ; P<0.05\right)$ in the perirhinal cortex (Fig. 5A). A similar trend is evident for the nontabulated laterality volumes in the entorhinal cortex (Fig. 5B) and hippocampus although the laterality differences failed to reach statistical significance $(P<0.11$ [Object $>$ Spatial]; $P<0.42$, respectively). The amygdala showed no significant differential lateralization during Object Encoding. Tabulated recognition laterality indices are depicted in Figure 5, C and D. Analysis of nontabulated laterality volume measures performed on the Recognition data also showed a significant Task by Hemisphere interaction $\left(F_{(1,13)}=\right.$ 7.004; $P<0.05$ ) in perirhinal cortex (Fig. 5C) that mirrored the Encoding effect; more active tissue in the left hemisphere for

Table 1. Laterality of significant group ANOVA effects

\begin{tabular}{lccc}
\hline & $\begin{array}{c}\text { Main effect } \\
\text { for Task }\end{array}$ & $\begin{array}{c}\text { Main effect } \\
\text { for Phase }\end{array}$ & $\begin{array}{c}\text { Interaction } \\
\text { Task } \times \text { Phase }\end{array}$ \\
\hline Entorhinal cortex & $\mathrm{L} / \mathrm{R}$ & $\mathrm{L}$ & $\mathrm{R}$ \\
Perirhinal cortex & $\mathrm{L}$ & Neither & $\mathrm{L} / \mathrm{R}$ \\
Anterior parahipp & $\mathrm{L}$ & $\mathrm{L}$ & $\mathrm{L} / \mathrm{R}$ \\
Posterior parahipp & $\mathrm{L} / \mathrm{R}$ & $\mathrm{L} / \mathrm{R}$ & $\mathrm{L}$ \\
Hippocampus & $\mathrm{L} / \mathrm{R}$ & $\mathrm{L} / \mathrm{R}$ & Neither \\
Amygdala & $\mathrm{L} / \mathrm{R}$ & $\mathrm{L} / \mathrm{R}$ & Neither \\
Fusiform gyrus & $\mathrm{L} / \mathrm{R}$ & $\mathrm{L} / \mathrm{R}$ & $\mathrm{L}$ \\
LOC & $\mathrm{L} / \mathrm{R}$ & $\mathrm{L} / \mathrm{R}$ & $\mathrm{L}$ \\
\hline
\end{tabular}

$P<0.05$; corrected.

(L) A significant effect in the left hemisphere; (R) a significant effect in the right hemisphere. 
Main Effect of Encoding $\underline{p}<0.05$ (corrected)

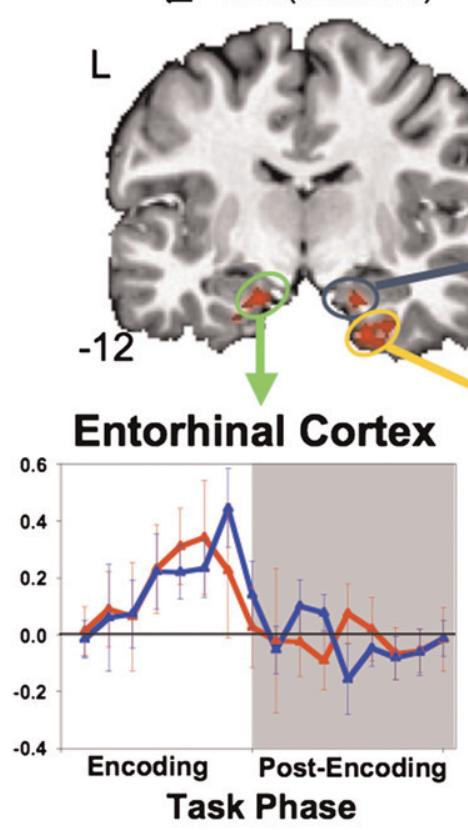

$\mathrm{R}$

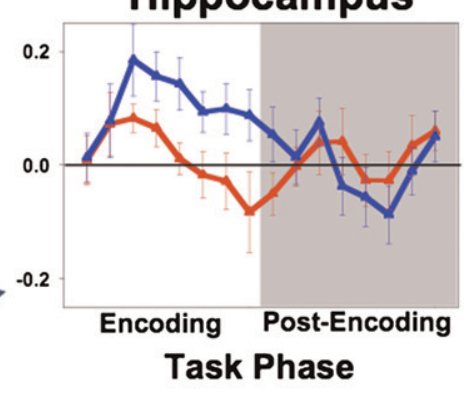

Perirhinal Cortex

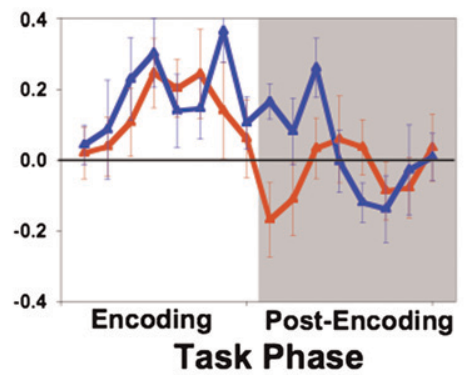

\section{Spatial Object} Task

Figure 2. The simple main effect for Encoding (both Object and Spatial) depicted in the coronal brain image. Red voxels represent areas showing increased BOLD responses for Encoding relative to baseline (post-recognition scramble). Graphs represent time-series data extracted from the entorhinal-, perirhinal-, and hippocampus-based active voxels from the Encoding main effect. Data are separated into their appropriate Encoding condition (blue = object; red = spatial).

Object encoding, more active tissue in the right hemisphere for Spatial encoding.

\section{Discussion}

Distinguishing the roles of specific MTL structures in long-term memory is a major goal in the study of memory. The hippocampus is the most studied of these regions as it serves a primary role in the encoding and time-limited retrieval of both object and spatial memory (Squire and Zola-Morgan 1991; Squire et al. 2004). The most prominent pathway into the hippocampus is the perforant pathway initiated in the entorhinal cortex. Anatomical connectivity of the hippocampus, parahippocampal cortex, perirhinal, and entorhinal cortices with the dorsal and ventral processing streams suggests that different neural circuits exist for processing object (ventral stream) and spatial (dorsal stream) memories (Suzuki and Amaral 1994, 2004; Suzuki et al. 1997; Burwell 2000; Munoz and Insausti 2005). Using the same stimuli and procedure as used in the present study, we previously reported that activity in the most anterior portion of the parahippocampal cortex, posterior to the perirhinal cortex, was greater for spatial than object encoding, while activity in the perirhinal cortex was equal for both object and spatial encoding. The present study confirmed and extended these findings using higher spatial resolution to reduce susceptibility-related signal dropout and improve tSNR in the MTL (Bellgowan et al. 2006). By carefully titrating our tasks (see Materials and Methods) to produce equivalent object and spatial recognition memory performance in the present as well as our previous study, we can rule out the possibility that the task-related differences in neural activity that we observed were contaminated by differences in task difficulty. However, because the composition of the recognition blocks in both tasks contained novel and repeated stimuli, encoding of the novel stimuli may have contaminated the task-related differences in neural activity seen during the recognition phases.

The perirhinal cortex is a major projection area to and from the entorhinal cortex (Mohedano-Moriano et al. 2007). If this connection is a component of the long-term memory system, it logically follows that the entorhinal cortex, like the perirhinal cortex, would participate in both forms of memory unless either form of information is carried through direct projections from the perirhinal cortex to the hippocampus. The present findings show that, like perirhinal cortex, the entorhinal cortex is active for both object and spatial encoding. Rather than complete lateralization, both regions showed biased hemispheric lateralization of function. Relative rather than complete lateralization suggests that task context biases lateralized processing of existing bilateral connections of the appropriate object or spatial processing circuitry.

This functional lateralization may be related to the traditional left/right MTL memory lateralization for verbal and nonverbal material, respectively, as documented by a large body of neuropsychological (e.g., Kimura 1963; Glosser et al. 1995; Bohbot et al. 2000; Kelley et al. 2002) and functional neuroimaging (e.g., Martin et al. 1997; Kelley et al. 1998; Martin 1999; O'Kane et al. 2005; Weber et al. 2007) evidence. For example, the left hemisphere bias for encoding object information in the present study could reflect the possibility that, although our stimuli were difficult to verbally encode, subjects may have
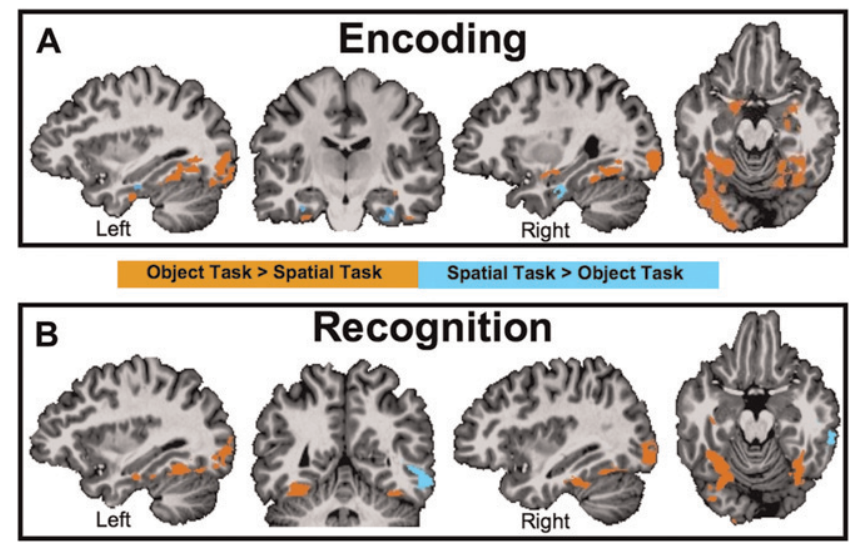

Figure 3. Map of contrast of means between the Object and Spatial conditions during both the $(A)$ Encoding and $(B)$ Recognition phases from the Group Mixed-Effects ANOVA. Orange areas represent significantly $(P$ $<0.05$ corrected) greater activation during the Object task relative to the Spatial task. Blue areas represent greater activation during the Spatial task relative to the Object task. In both the coronal and axial slices, the left hemisphere is on the left and the right hemisphere is on the right. 


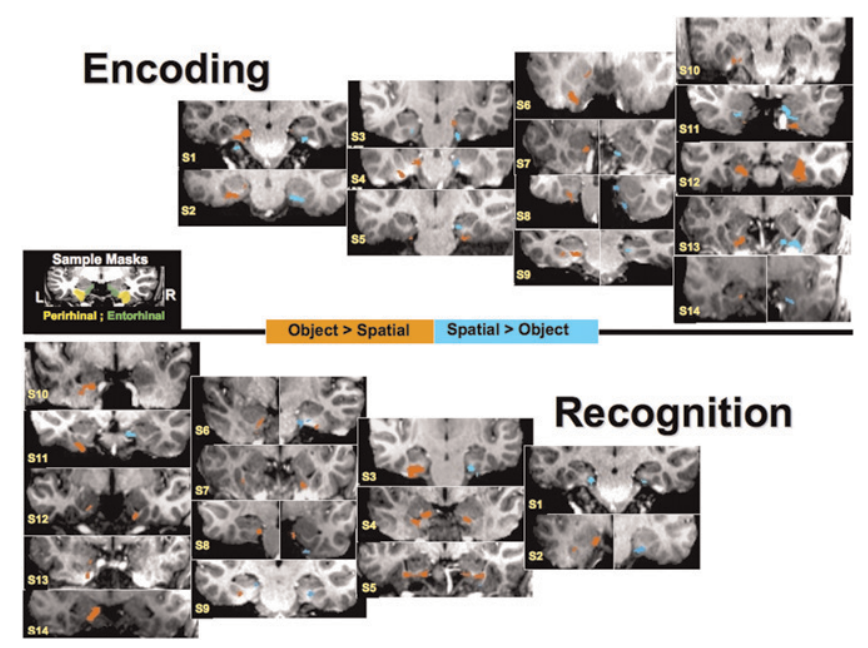

Figure 4. Individual activation maps for the Object vs. Spatial contrast during both Encoding and Recognition. Orange voxels represent areas showing Object $>$ Spatial at a $P<0.05$ corrected value whereas blue voxels show areas where Spatial $>$ Object at that same threshold. The middle figure depicts a single subject's anatomical mask for the entorhinal and perirhinal cortex. S1-S14 are the representative subject numbers. Bisected individual images depict the same statistical comparison at different slice locations. In each coronal slice, the left hemisphere is on the left and the right hemisphere is on the right.

nevertheless assigned verbal attributes to the fractals during the objects task (e.g., color, spikiness, etc.) to aid memory. In contrast, during the spatial task, the subjects were required to remember only the spatial location, thus biasing memory processing to the right hemisphere.

There is currently considerable debate and controversy concerning how best to characterize the distinct roles that different regions of the MTL play in human memory function. In the context of this debate, Squire and colleagues have recently reintroduced a proposal by Underwood (1969) that memories can be conceptualized as a configural set of stimulus attributes such as verbal associates, stimulus frequency, and temporal/ spatial components, and that these components may be processed differentially within the MTL (Squire et al. 2007). Our findings are consistent with this view. Parahippocampal cortex, known to receive a stronger projection from the dorsal than ventral processing stream, showed a spatial memory bias (see also Buffalo et al. 2006), whereas perirhinal and entorhinal cortices, known to receive inputs from both processing streams, are involved in both object and spatial memory. Moreover, these regions show lateralized functioning with a right hemisphere bias for spatial and a left hemisphere bias for object encoding and recognition memory.

\section{Materials and Methods}

\section{Participants}

Twenty (10 female) right-handed, native English-speaking adults (ages 22-34) participated for $\sim 2 \mathrm{~h}$ in this experiment. All subjects signed informed consent in compliance with the IRB at $\mathrm{NIH}$ and were monetarily compensated for their participation. Six subjects were removed from analysis owing to uncorrectable movement artifacts (two) or uncorrectable transient imaging hardware problems (four).

\section{Tasks and stimuli}

Participants performed alternating spatial and object memory tasks known to require participation of the MTL (Buffalo et al. 1998, 2006). Buffalo et al. (2006) previously performed a series of strictly behavioral tests, no scanning, using this same task in order to equate the recognition performance of the Object and Spatial memory task. To accomplish this, Buffalo et al. (2006) titrated the number of possible locations in the Spatial memory task so that recognition performances would be equivalent. The final number of possible locations in the Spatial task was 12.

Both tasks consisted of a sequence of four 18-sec blocks including an Encoding block, a Post-Encoding delay, a Recognition memory block, and a Post-Recognition delay interval that served as a baseline (see Fig. 1A). Two of these four-block sequences were repeated for both the Spatial and Object memory tasks during each functional scan. Stimulus presentation parameters were equivalent for both memory tasks. Stimuli for Encoding and Recognition were unique, relatively nonverbalizeable, colored fractal patterns (Miyashita et al. 1991). During the delay blocks, a single scrambled version of a fractal pattern was repeatedly presented. During each block, fractal patterns were presented for $2500 \mathrm{msec}$ with a 500msec interstimulus interval (ISI).

Prior to scanning, subjects were instructed to memorize fractals and ignore their location for the Object memory task, and to memorize their location while ignoring their identity during the Spatial memory task. During the Encoding and Recognition blocks, the fractals were presented in any of 16 locations distributed throughout the screen. During scanning, subjects were cued to the task with an instruction screen that read "Memorize the Object" before the Object Encoding phase, "Watch the Picture" prior to the delay periods, and "Recall the Object" prior to the Object Recognition phase. The instructions during the Spatial location task were structured identically except that the cues read "Memorize the Location" and "Recall the Location" prior to the Encoding and Recognition phases, respectively. During Recognition blocks, subjects made Old/New forced choice
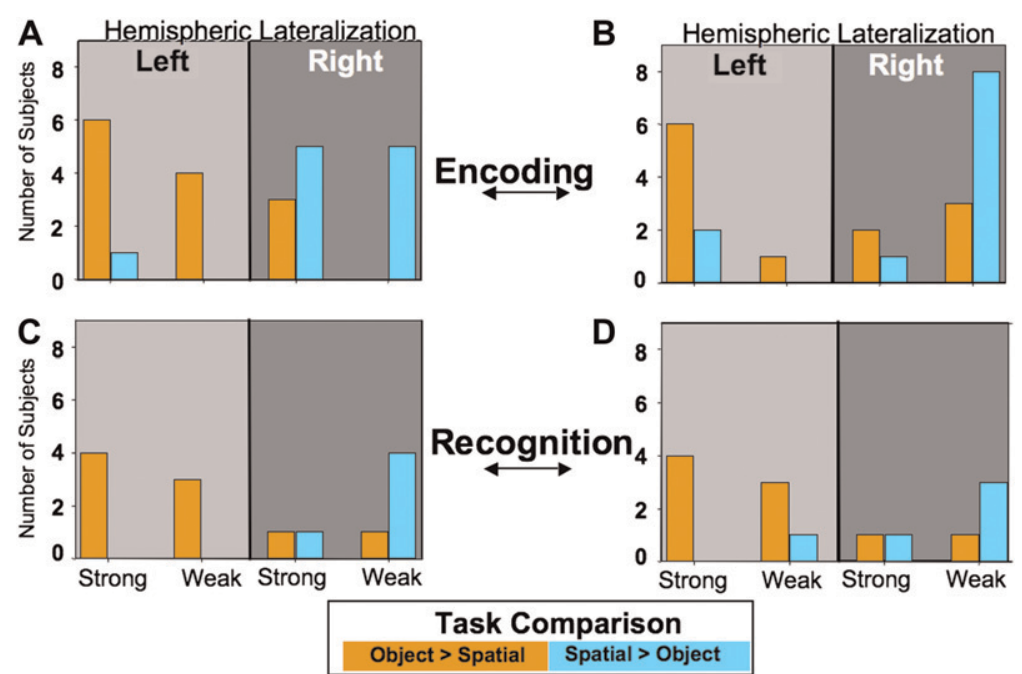

Figure 5. Histograms depicting the distribution among all subjects of the tabulated (Strong or Weak) laterality indices for the Object > Spatial contrast (orange bars) and Spatial > Object contrast (blue bars) for both the $(A)$ perirhinal and $(B)$ entorhinal cortex. $(A, B)$ The distributions for both the perirhinal and entorhinal cortex, respectively, during encoding. $(C, D)$ The distributions during recognition performance for the perirhinal and entorhinal cortex, respectively. The left portions of each graph (lighter gray) are tallies that resulted in left hemisphere lateralization, and conversely the right portions of each graph (darker gray) are tallies that resulted in right hemisphere lateralization. 
decisions to each stimulus presentation. Half of stimuli were novel, and half were repeats (targets) from the Encoding block (either the same object or same location as studied previously). During Object Recognition blocks, the target stimuli were always in different locations from what had been presented during Object Encoding. During Spatial Recognition blocks, the target stimuli were always different objects from what had been presented during Spatial Encoding. The order of the memory task type (Object or Spatial memory) within a functional scan was counterbalanced across subjects.

\section{Magnetic resonance imaging}

Scanning was performed on a 3-T General Electric (GE) Signa VH/3 whole body MRI scanner. This scanner was equipped with a homebuilt, scalable multichannel MRI digital receiver that allowed use of a head receive-only 16-element surface coil array for signal detection (Bodurka et al. 2004). Sixteen axial slices were aligned to cover most of the temporal lobe (see Fig. 1B). Functional images were collected using a single-shot, full k-space gradient-echo echoplanar (EPI) sequence $(\mathrm{TR}=2000 \mathrm{msec}, \mathrm{TE}=40 \mathrm{msec}$, flip angle = $90^{\circ}, \mathrm{FOV}=200 \mathrm{~mm}$, and in plane resolution $3.125 \times 3.125 \mathrm{~mm}^{2}$, slice thickness of $2 \mathrm{~mm}$, and 132 functional volumes). EPI data acquisition was done in parallel across all 16 channels. The resulting 16 images for each slice and each time point were then reconstructed from raw data and combined into a single magnitude combined (square root of the sums squared) image. Four functional scanning series were performed on each subject for a total of eight Spatial and eight Object task cycles. A whole brain high-resolution (FOV $=220 \mathrm{~mm}, 1.2 \mathrm{~mm}$ slice thickness, and in plane resolution $1.0 \times 1.0 \mathrm{~mm}^{2}$ ) anatomical image was acquired using a Magnetization Prepared Rapid Acquisition Gradient-Echo imaging sequence (MPRAGE).

All analyses were performed using AFNI (Cox 1996). Following removal of the first four volumes of each series, the four series were concatenated, motion corrected, blurred with a 2-mm RMS filter, and mean standardized to a value of 100 . Individual time series were submitted to a voxelwise multiple regression analysis with six regressors of interest consisting of factorial components of Task (Object, Spatial) and Task Phase (Encoding, Delay, and Recognition). Six motion correction regressors were included as regressors of no interest. The regressors of interest were convolved with a Gamma-variate model (Cohen 1997) to incorporate the hemodynamic delay into the model. Post-Recognition Delay was set as the baseline in this regression analysis. Anatomical regions of interest were created for the entorhinal and perirhinal cortex following the procedure described by Insausti et al. (1998). Additional, anatomical regions of interest mask were created for the hippocampus and amygdala, and parahippocampal cortex (Pruessner et al. 2002). Both individual and group statistical maps were corrected for multiple comparisons using a statistical and volume threshold (Forman et al. 1995) (http://afni.nimh.nih.gov/ afni/doc/manual/AlphaSim), where volume correction was calculated based on the total volume of the combined anatomical ROIs (bilateral entorhinal cortex, perirhinal cortex, hippocampus, parahippocampal cortex, and amygdala) and a voxelwise statistical threshold set to $P<0.01$.

Object (Object $>$ Spatial) and Spatial (Spatial > Object) task laterality indices were computed for both the Encoding and Recognition phases in the perirhinal and entorhinal cortex and the hippocampus for each subject. The metric for this index was the volume of active tissue surviving a corrected $P<0.05$ for the contrast of means between the Object and Spatial task for each respective phase within each anatomically defined region. These volumes were then converted to a laterality index (LI) using the following formula: $\mathrm{LI}=(\mathrm{L}-\mathrm{R}) /(\mathrm{L}+\mathrm{R})$, where $\mathrm{L}$ depicts the volume of active tissue in the left hemisphere and $\mathrm{R}$ depicts the volume of active tissue in the right hemisphere. This index yielded both positive and negative values that reflect degree of left and right hemisphere lateralization, respectively. LIs were categorized into either "Strong" $(|\mathrm{LI}|>0.5)$ or "Weak" $(|\mathrm{LI}|<0.5)$. Both categories were then tabulated for left and right lateralization within each region.
The group analysis consisted of a voxelwise Mixed-Effects group ANOVA with subjects as a random factor and Task (Object, Spatial) and Task Phase (Encode, Delay, Recognition) as fixed factors. The resulting statistical maps were corrected for multiple comparisons $(P<0.05)$ using the cluster volume by statistical threshold correction method described above.

\section{Acknowledgments}

We thank Paula Rowser and Alda Ottley for their assistance in data collection and W. Kyle Simmons for insightful comments. This research was supported by the Intramural Research Program of the $\mathrm{NIMH}, \mathrm{NIH}$.

\section{References}

Bellgowan PSF, Binder JR, Swanson SJ, Hammeke TA, Springer JA, Frost JA Mueller WM, Morris GL. 1998. Side of seizure focus predicts left medial temporal lobe activation during verbal encoding. Neurology 51: 479484.

Bellgowan PSF, Bandettini PA, van Gelderen P, Martin A, Bodurka J. 2006. Improved BOLD detection in the medial temporal region using parallel imaging and voxel volume reduction. Neuroimage 29: 12441251.

Bodurka J, Ledden PJ, van Gelderen P, Chu R, de Zwart J, Morris D, Duyn JH. 2004. Scalable multichannel MRI data acquisition system. Magn Reson Med 51: 165-171.

Bohbot VD, Allen JJB, Nadel L. 2000. Memory deficits characterized by patterns of lesions to the hippocampus and parahippocampal cortex. Ann N Y Acad Sci 911: 355-368.

Buffalo EA, Reber PJ, Squire LR. 1998. The human perirhinal cortex and recognition memory. Hippocampus 8: 330-339.

Buffalo EA, Bellgowan PSF, Martin A. 2006. Distinct roles for medial temporal lobe structures in memory for objects and their locations. Learn Mem 13: 638-643.

Burwell RD. 2000. The parahippocampal region: Corticocortical connectivity. Ann N Y Acad Sci 911: 25-42.

Cohen M. 1997. Parametric analysis of fMRI data using linear systems methods. Neuroimage 6: 93-103.

Cox RW. 1996. AFNI: Software for analysis and visualization of functional magnetic resonance neuroimages. Comput Biomed Res 29: 162-173.

Forman SD, Cohen JD, Fitzgerald M, Eddy WF, Mintun MA, Noll DC. 1995 Improved assessment of significant activation in functional magneticresonance-imaging (fMRI) - use of a cluster-size threshold. Magn Reson Med 33: 636-647.

Fyhn M, Molden S, Witter MP, Moser EI, Moser MB. 2004. Spatial representation in the entorhinal cortex. Science 305: 1258-1264.

Glosser G, Saykin AJ, Deutsch GK, O'Connor MJ, Sperling MR. 1995. Neura organization of material-specific memory functions in temporal-lobe epilepsy patients as assessed by the intracarotid amobarbital test. Neuropsychology 9: 449-456.

Hafting T, Fyhn M, Molden S, Moser MB, Moser EI. 2005. Microstructure of a spatial map in the entorhinal cortex. Nature 436: 801-806.

Hargreaves EL, Rao G, Lee I, Knierim JJ. 2005. Major dissociation between medial and lateral entorhinal input to dorsal hippocampus. Science 308: 1792-1794.

Insausti R, Juottonen K, Soininen H, Insausti AM, Partanen K, Vainio P, Laakso MP, Pitkanen A. 1998. MR volumetric analysis of the human entorhinal, perirhinal, and temporopolar cortices. AJNR Am J Neuroradiol 19: 659-671.

Jones-Gotman M, Zatorre RJ, Olivier A, Andermann F, Cendes F, Staunton H, McMackin D, Siegel AM, Wieser HG. 1997. Learning and retention of words and designs following excision from medial or lateral temporallobe structures. Neuopsychologia 35: 963-973.

Kelley WM, Miezin FM, McDermott KB, Buckner RL, Raichle ME, Cohen NJ, Ollinger JM, Akbudak E, Conturo TE, Snyder AZ, et al. 1998. Hemispheric specialization in human dorsal frontal cortex and medial temporal lobe for verbal and nonverbal memory encoding. Neuron 20: 927-936.

Kelley WM, Ojemann JG, Wetzel RD, Derdeyn CP, Moran CJ, Cross DT, Dowling JL, Miller JW, Petersen SE. 2002. Wada testing reveals frontal lateralization for the memorization of words and faces. J Cogn Neurosci 14: $116-125$.

Kennepohl S, Sziklas V, Garver KE, Wagner DD, Jones-Gotman M. 2007. Memory and the medial temporal lobe: Hemispheric specialization reconsidered. Neuroimage 36: 969-978.

Kerr K, Agster K, Furtak S, Burwell R. 2007. Functional neuroanatomy of the parahippocampal region: The lateral and medial entorhinal areas. Hippocampus 17: 697-708. 
Kimura D. 1963. Right temporal-lobe damage-perception of unfamiliar stimuli after damage. Arch Neurol 8: 264-271.

Martin A. 1999. Automatic activation of the medial temporal lobe during encoding: Lateralized influences of meaning and novelty. Hippocampus 9: $62-70$.

Martin A, Wiggs CL, Weisberg J. 1997. Modulation of human medial temporal lobe activity by form, meaning, and experience. Hippocampus 7: 587-593.

Miller MI, Beg MF, Ceritoglu C, Stark C. 2005. Increasing the power of functional maps of the medial temporal lobe by using large deformation diffeomorphic mapping. Proc Natl Acad Sci 102: 9685-9690.

Milner B. 1972. Disorders of learning and memory after temporal-lobe lesions in man. Clin Neurosurg 19: 421-446.

Miyashita Y, Higuchi S, Sakai K, Masui N. 1991. Generation of fractal patterns for probing the visual memory. Neurosci Res 12: 307-311.

Mohedano-Moriano A, Pro-Sistiaga P, Arroyo-Jimenez MM, ArtachoPerula E, Insausti AM, Marcos P, Cebada-Sanchez S, Martinez-Ruiz J, Munoz M, Blaizot X, et al. 2007. Topographical and laminar distribution of cortical input to the monkey entorhinal cortex. J Anat 211: $250-260$.

Moscovitch C, Kapur S, Kohler S, Houle S. 1995. Distinct neural correlates of visual long-term memory for spatial location and object identity: A positron emission tomography study in humans. Proc Natl Acad Sci 92: 3721-3725.

Munoz M, Insausti R. 2005. Cortical efferents of the entorhinal cortex and the adjacent parahippocampal region in the monkey (Macaca fascicularis). Eur J Neurosci 22: 1368-1388.

O'Kane G, Insler RZ, Wagner AD. 2005. Conceptual and perceptual novelty effects in human medial temporal cortex. Hippocampus 15: 326-332.

Pruessner JC, Kohler S, Crane J, Pruessner M, Lord C, Byrne A, Kabani N, Collins DL, Evans AC. 2002. Volumetry of temporopolar, perirhinal, entorhinal and parahippocampal cortex from high-resolution MR images: Considering the variability of the collateral sulcus. Cereb Cortex 12: $1342-1353$.

Saleem KS, Tanaka K. 1996. Divergent projections from the anterior inferotemporal area TE to the perirhinal and entorhinal cortices in the macaque monkey. J Neurosci 16: 4757-4775.

Seffenach H, Witter M, Moser M, Moser E. 2005. Spatial memory in the rat requires the dorsolateral band of the entorhinal cortex. Neuron 45: 301313 .

Sommer T, Rose M, Glascher J, Wolbers T, Buchel C. 2005. Dissociable contributions within the medial temporal lobe to encoding of objectlocation associations. Learn Mem 12: 343-351.

Squire LR, Zola-Morgan S. 1991. The medial temporal-lobe memory system. Science 253: 1380-1386.

Squire LR, Stark CEL, Clark RE. 2004. The medial temporal lobe. Annu Rev Neurosci 27: 279-306.

Squire LR, Wixted JT, Clark RE. 2007. Recognition memory and the medial temporal lobe: A new perspective. Nat Neurosci Rev 8: 872-883.

Suzuki WA, Amaral DG. 1994. Perirhinal and parahippocampal cortices of the macaque monkey-cortical afferents. J Comp Neurol 350: 497533.

Suzuki WA, Amaral DG. 2004. Functional neuroanatomy of the medial temporal lobe memory system. Cortex 40: 220-222.

Suzuki WA, Miller EK, Desimone R. 1997. Object and place memory in the macaque entorhinal cortex. J Neurophysiol 78: 1062-1081.

Underwood BJ. 1969. Attributes of memory. Psychol Rev 76: 559-573.

Weber B, Fliessbach K, Lange N, Lange N, Kugler F, Elger CE. 2007. Materialspecific memory processing is related to language dominance. Neuroimage 37: 611-617.

Received January 30, 2009; accepted in revised form April 13, 2009. 


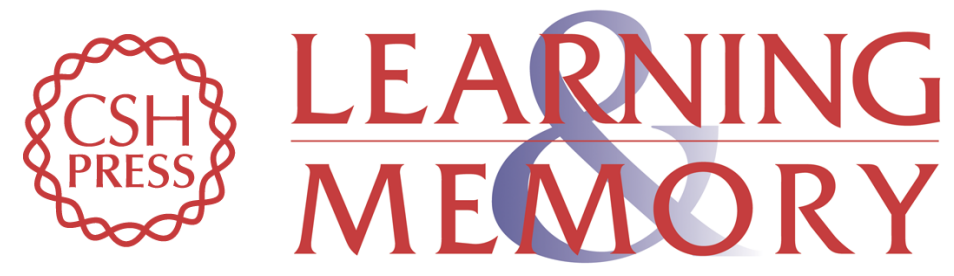

\section{Lateralized spatial and object memory encoding in entorhinal and perirhinal cortices}

Patrick S.F. Bellgowan, Elizabeth A. Buffalo, Jerzy Bodurka, et al.

Learn. Mem. 2009, 16:

Access the most recent version at doi:10.1101//m.1357309

References This article cites 42 articles, 9 of which can be accessed free at:

http://learnmem.cshlp.org/content/16/7/433.full.html\#ref-list-1

License Freely available online through the Learning \& Memory Open Access option.

Email Alerting Receive free email alerts when new articles cite this article - sign up in the box at the Service top right corner of the article or click here. 